\title{
Wave Processes and Mass Transfer on the Copper-Stainless Steel Interface under Solid Phase Bonding by High-Temperature Rolling
}

\author{
Boris V. Borts, Aleksander A. Parkhomenko, Igor O. Vorobyov, Alexander A. Lopata \\ National Science Center, Kharkov Institute of Physics \& Technology, Kharkov, Ukraine \\ Email: borts@kipt.kharkov.ua
}

How to cite this paper: Borts, B.V., Parkhomenko, A.A., Vorobyov, I.O. and Lopata, A.A. (2018) Wave Processes and Mass Transfer on the Copper-Stainless Steel Interface under Solid Phase Bonding by High-Temperature Rolling. Open Journal of Metal, 8, 67-74.

https://doi.org/10.4236/ojmetal.2018.84005

Received: November 13, 2018

Accepted: December 26, 201

Published: December 29, 2018

Copyright $\odot 2018$ by author(s) and Scientific Research Publishing Inc. This work is licensed under the Creative Commons Attribution International License (CC BY 4.0).

http://creativecommons.org/licenses/by/4.0/

\begin{abstract}
The paper presents the study of hierarchy of deformation wave-processes from nano- to macro-structural level, which takes place in dissimilar materials, bonded by high-temperature vacuum rolling in solid phase. The focus was on the processes that occur on the interface of the bonded materials: mass trasfer of impurities and alloying elements stimulated by deformation, the study of nano- and micro-hardness.
\end{abstract}

\section{Keywords}

Vacuum Hot Rolling, Solid Phase, Interface, Cupper-Stainless Steel, Microscopy, Micro-Hardness, Nano-Hardness, Wave Processes, Scaling

\section{Introduction}

In the last years, NSC KIPT has studied the areas of interface of $\mathrm{Cu}-\mathrm{Nb}$ and $\mathrm{Cu}-\mathrm{Pb}$ [1] [2] systems bonded by high-temperature vacuum rolling in solid phase. On results of this study the following deformation nano-deffects and processes were discovered, indicating the presence of the hydro-dinamic and rotation modes of deformation: "wave front" and dynamic nano-crystallisation, nano-dipoles of partial disclinations, and nano-dipoles of rotation disclinations.

The purpose of this work is to analyze all sequences of structural levels of wave processes that occur on macro-, micro- and nano-levels, on the interfaces $\mathrm{Cu}$-stainless steel, bonded by high-temperature rolling in vacuum, considering the processes of mass-transfer in the areas of interface.

\section{Methods}

Solid-phase bonding of these materials was performed on the rolling machine 
ДУО-170 [3] in general package using transition layers-Ststeel-Cu-Nb-Ti under temperature $950^{\circ} \mathrm{C}-1000^{\circ} \mathrm{C}$ in vacuum. Rolling deformation constituted $27 \%$ $30 \%$. Foils with thickness $0.15-0.2 \mathrm{~mm}$ were prepared from the package, bonded in solid phase. Samples for electron microscopic study were made from these foils. High-resolution electron microscopy (microscope JEM-2100F) was used for analyses of processes that occur on the interface of materials, bonded in solid phase by high-temperature vacuum rolling.

Metallographic study was performed on optical microscope Olympus GX-51 and raster electronic microscope ZEISS-EVO-50, equipped with energy dispersion analyzer INCA-450. Study of changes of micro-hardness and nano-hardness through the interface of stainless-steel-copper was performed on micro-hardness testing machine LECOLM-700 and nano-hardness testing machine NANOG200, manufactured in the USA in compliance with to the rules of international standard ISO 14577.

\section{Results and Discussions}

Macro-structural level. Surface waves of Kelvin-Helmholtz type with wave lengths $100-800 \mathrm{mcm}$ [4] were observed on the interfaces of copper-stainless-steel, generated by high-temperature rolling. Examples of such processes are shown in Figure 1. It should be noted that such well-ordered wave structures are observed in the same materials under absolutely different conditions of solid-phase bonding, for example under explosion welding [5].

Micro-structural level. Example of micro-structural (dislocation) level event is shown in Figure 2.

Figure 3 and Figure 4 demonstrate change of micro-hardness and nano-hardness relative to the interface of two metals. The following common patterns can be noted: less strength material-copper when reaching the interface is strengthening and more strength material-stainless steel-is softening. The

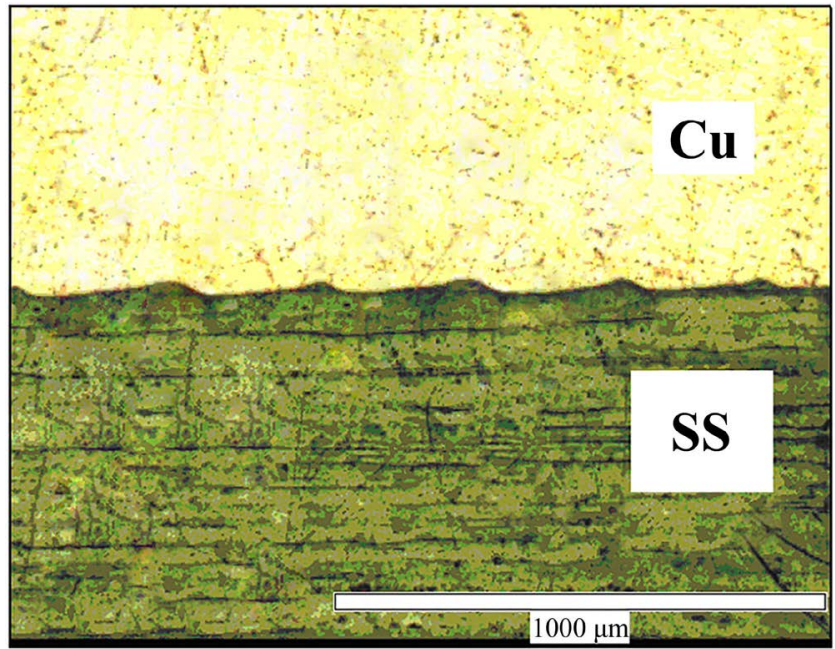

Figure 1. Wave processes with period about $300 \mathrm{mcm}$ on the interface of copper and stainless steel, bonded by high-temperature rolling in vacuum. 


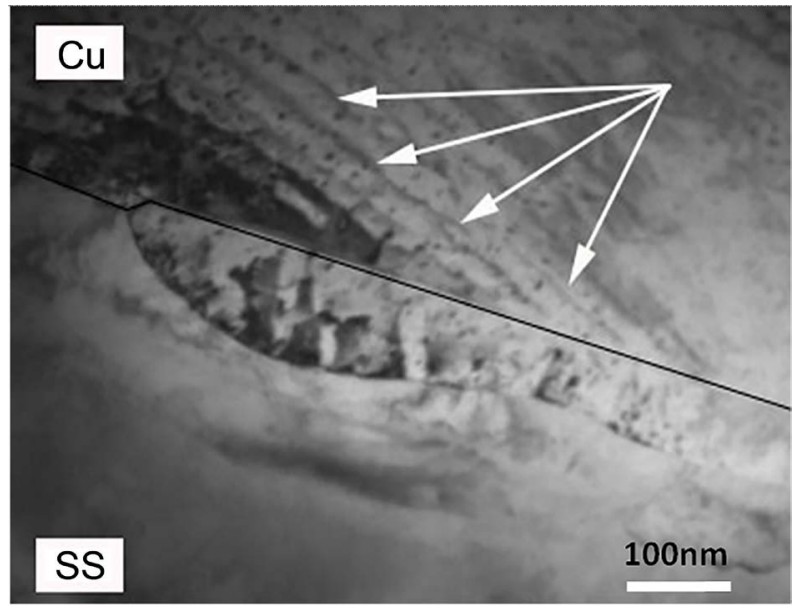

Figure 2. Example of the event of micro-structural level: dislocation mass-transfer. Formation of quasi-periodic dislocation structure (showed by arrows).

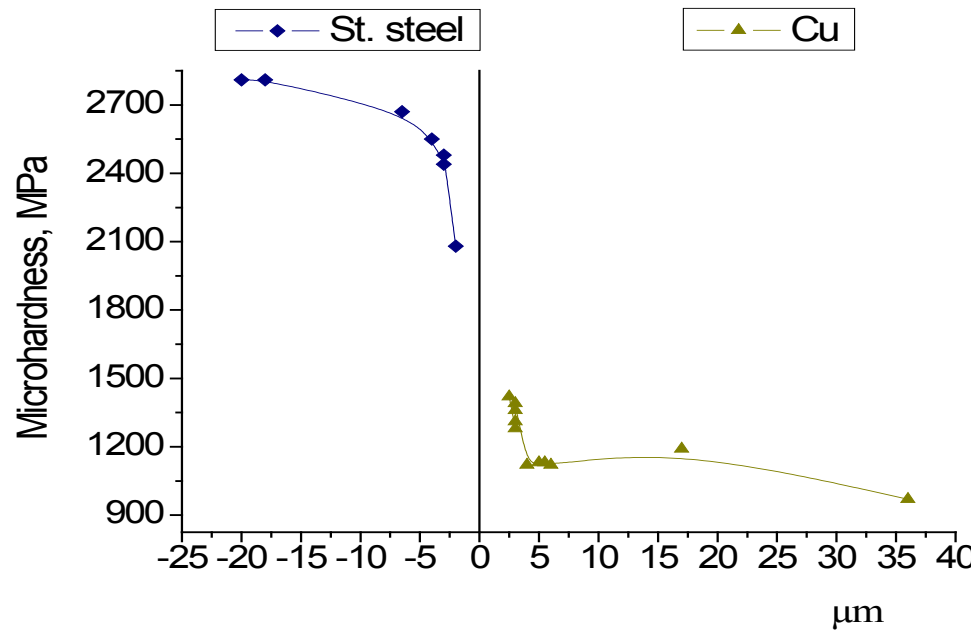

Figure 3. Distribution of micro-hardness relatively to SS-Cu interface.

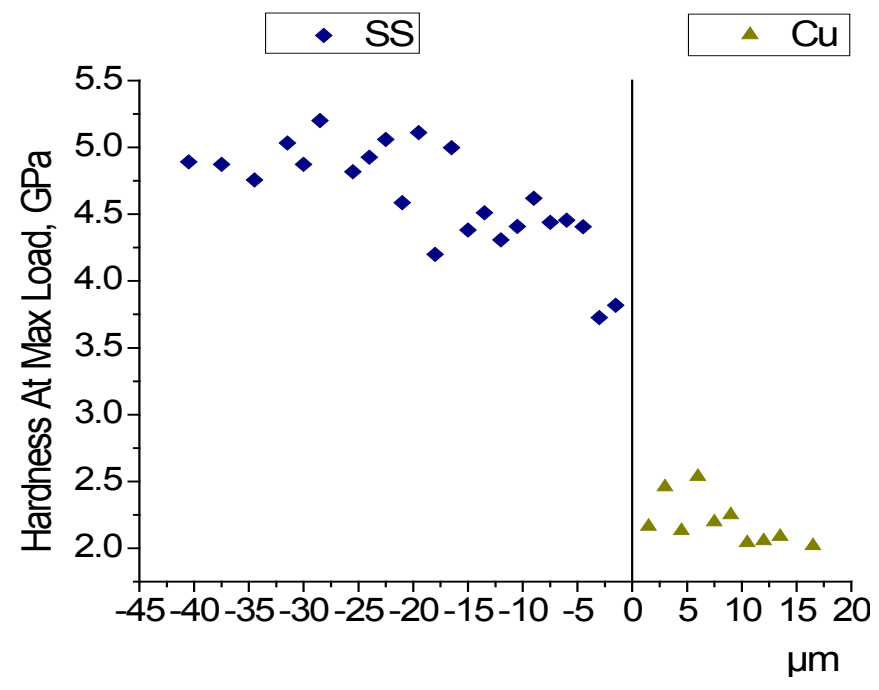

Figure 4. Change of the nano-hardness from the interface (demonstrated by "zero" line) $\mathrm{Cu}-08 \mathrm{X} 18 \mathrm{H} 10 \mathrm{~T}$. 
same tendencies are observed in the similar studies performed on $\mathrm{Cu}-\mathrm{Pb}$ [2] system.

Results of the micro-X-ray-spectral analyses, showed in Figure 5, explain the results shown above. Essential mixture of components of copper and steel on both sides of the interface is observed. At this, the penetration depth of copper in steel exceeds considerably the penetration depth of chrome, nickel, and iron in copper. Again it should be noted, that the same tendency-deeper penetration of more low-melting metal was indicated in the recent paper [2] on $\mathrm{Cu}-\mathrm{Pb}$ system.

Structural studies provide evidence of dislocation-stimulating diffusion of atoms of alloying elements from steel $08 \mathrm{X} 18 \mathrm{H} 10 \mathrm{~T}$ into copper during the high-temperature rolling in vacuum. It is confirmed by coincidence of visible in figure dislocation patterns and geometry of structures, formed by atoms diffusing from steel (compare Figure 6 and Figure 8). The most interesting was observation of the fact that atoms of alloying elements diffuse on deformation structure in the form of complexes, clusters, containing $3-5$ atoms. Results of the analyses of atoms distribution into the depth of interface constitute the evidence of this (Figure 7 and Figure 8). This can be the evidence of collective character of non-equilibrium diffusion in conditions of high-temperature deformation of bonds. It can be assumed that there is existence of peculiar cooperative dislocation mechanism of atoms movement under impact of stress gradient, under conditions of high super-saturation of copper with deformation and thermal defects. As to the chrome, formation of clusters enriched with it, with add-mixtures and their transfer by dislocations to the interface in $\mathrm{Fe}-\mathrm{Cr}$ alloys and steels, was experimentally determined in the paper [6] using the Atome Probe Tomography.

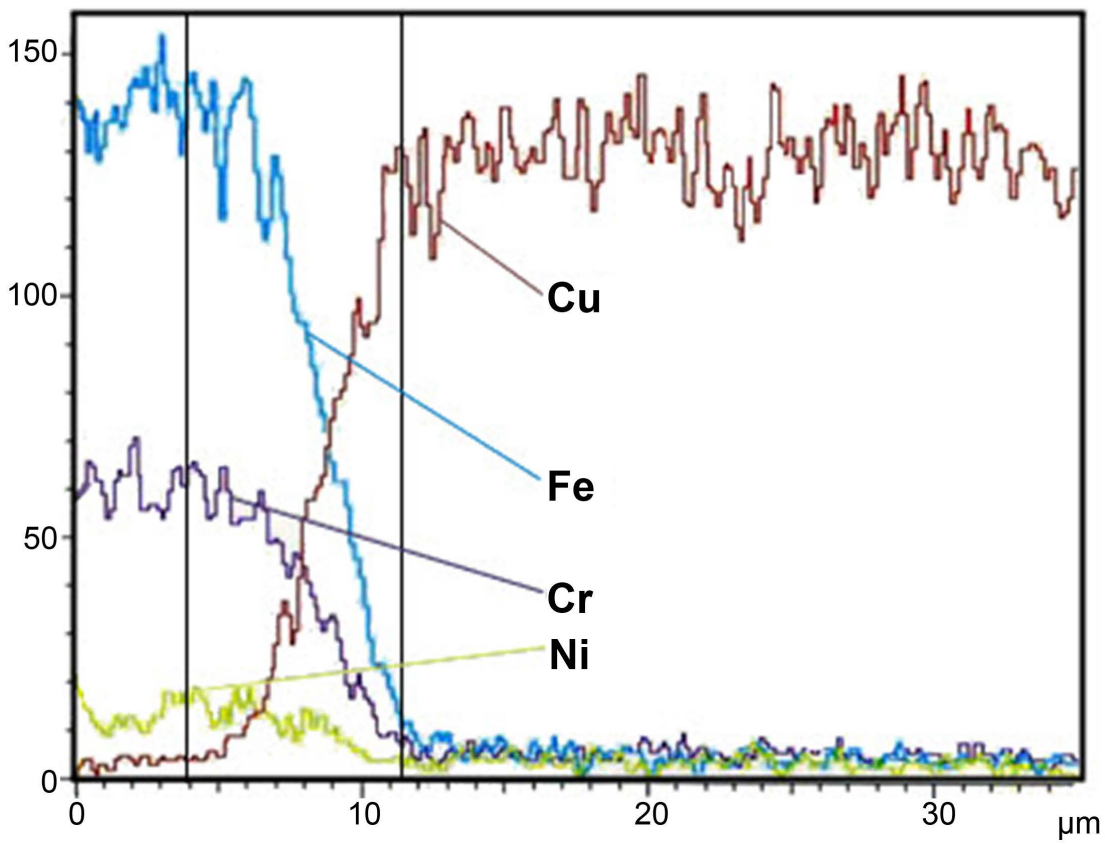

Figure 5. Results of micro-X-ray-spectral analyses of elements distribution close to the interface of steel-copper. 


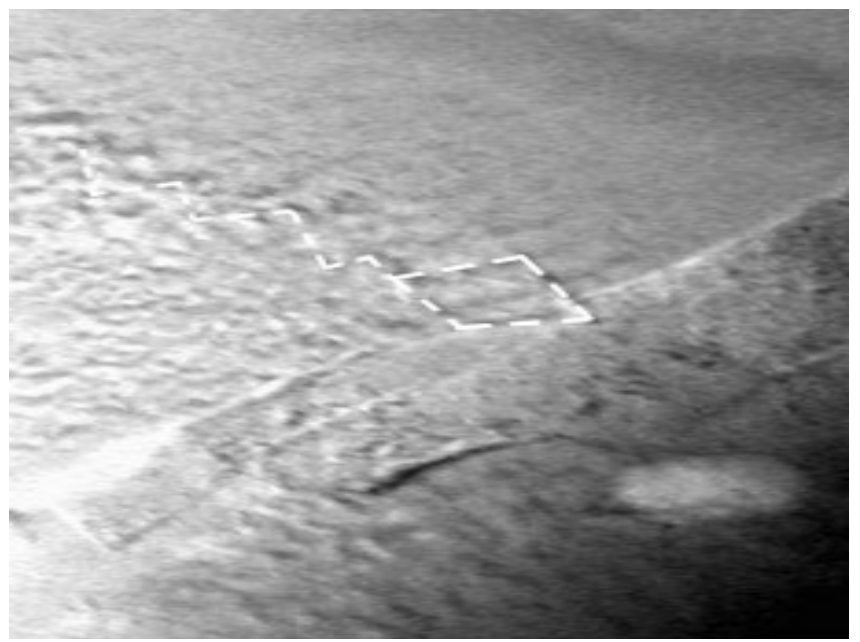

Figure 6. Electronic image of the interface structure steel-copper. The broken line demonstrates distribution of dislocations in the form of net structure.

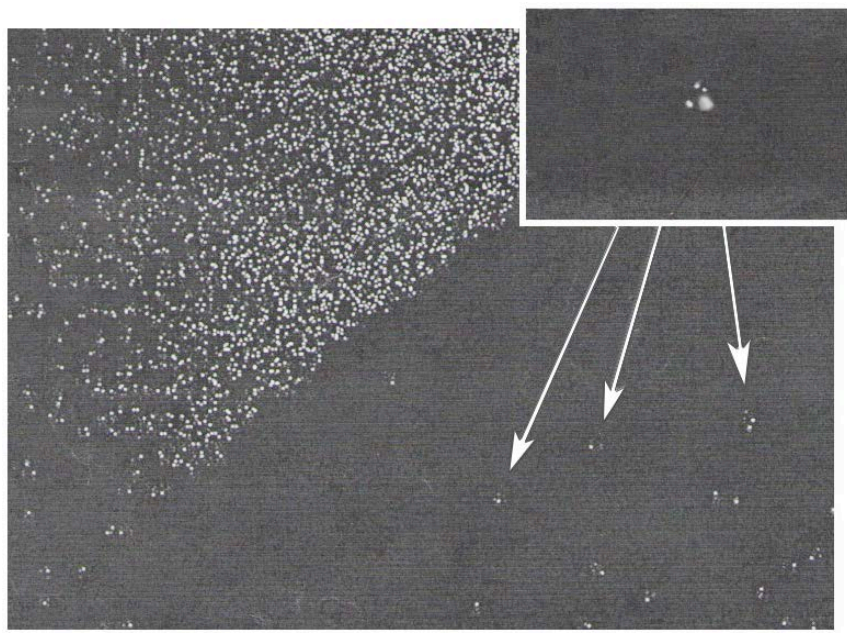

Figure 7. Distribution of copper atoms in the depth of stainless steel. The insert demonstrates the diffusing complex of atoms.

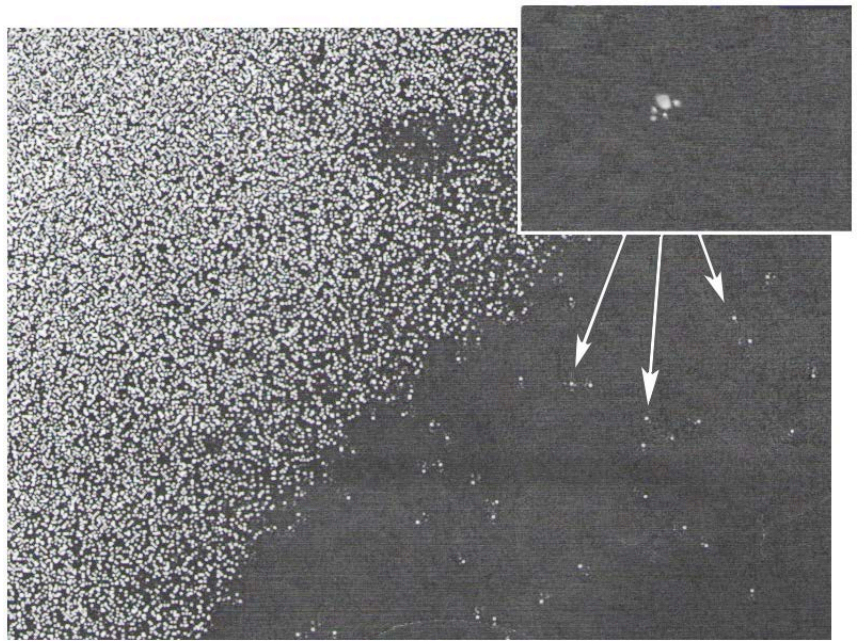

Figure 8. Distribution of atoms of chrome, withdrawn from steel in copper. 
Effect of anomalous mass-transfer in the deformed with high speeds (or under conditions of impulsive loads) materials was in the focus of metal physicist [7] [8] [9]. It was determined that atoms mobility depends on the nature of diffusion element. Increase of atoms mobility on $1-2$ order is observed with the growth of deformation level for the case of substitution atoms (as in the system Cu-stainless steel)

This corresponds to the main approach of physical meso-mechanics, according to which, under conditions of high excitation of the non-equilibrium structural condition caused by action of concentrated fluxes of energy, new allowed structural states occur in the interstitials of the crystal lattice [10]. According to the works of these authors, on $\mathrm{N}$ atoms of the solid body under conditions of excitation, there are $2 \mathrm{~N}$ of structural conditions, half of which turns to be vacant. So called atom vacancy configurations occur in the material, which can stipulate even the hydro-dynamic mass transfer, with collective displacement of atoms in the lattice.

Structure of the interface of the bonded materials was studied using high-resolution microscopy.

As it can be seen from Figure 9, even high-resolution electron microscopy provides limited opportunity for determination of the location of the materials interface, necessary for analysis. At the same time "Fourier purification" allows not only to determine the location of the interface, but also identifies a number of peculiarities of its structure.

Dislocation defects, limiting the spatial location of the interface are marked with arrows in Figure 9. The fact that their quantity from the lower side of the

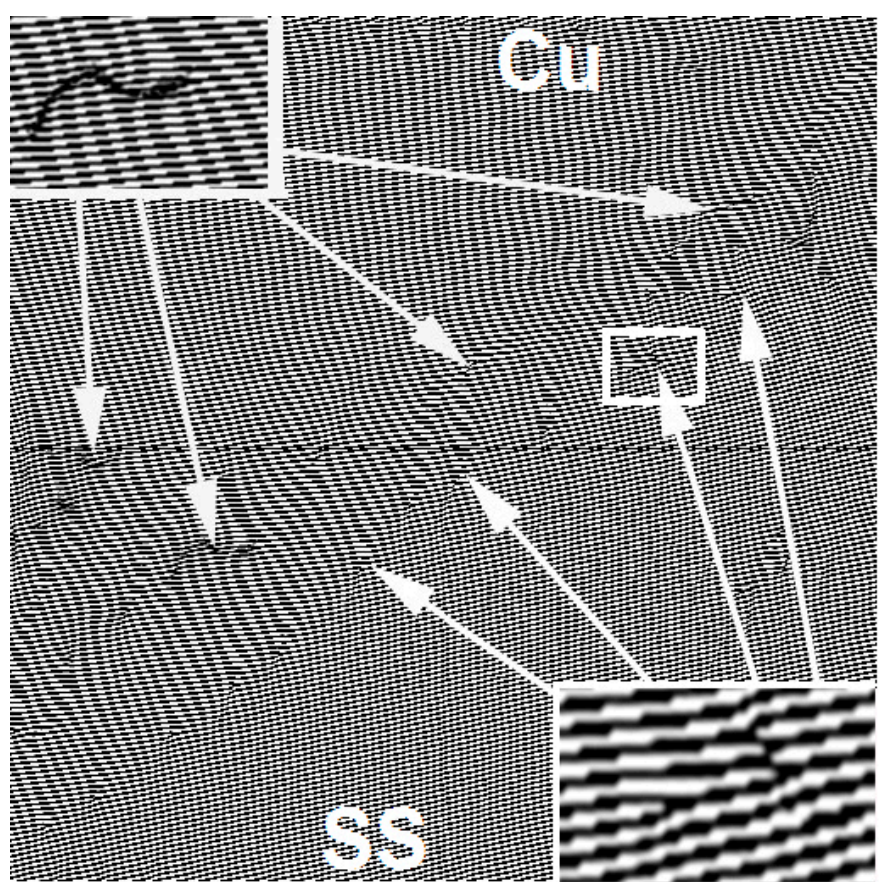

Figure 9. Structure of transition area of bimetal SS-copper. Picture obtained by transmission electron microscopy of high resolution and "Fourier purification". 
interface is much larger, than from the upper site, indicates on the direction of mass-transfer (motion of the interface-top-down). The defects of the dislocation type form the non-completed, irregular dislocation wall with Burgers vector, comparable on module with lattice parameters. Just like for the case of niobium-copper [11], the interface (in the most part of its length) is coherent with matrix excluding the area occupied by the mentioned above network.

Similar nano-defects were also discovered recently when using the high-resolution electronic microscopy in the alloy $\mathrm{Ti}-\mathrm{Nb}$ structure [12], but after large values of severe plastic deformation $(e=9.3)$.

\section{Conclusions}

1) Passing of the surface waves of Kelvin-Helmholtz type with wave lengths $100-800 \mathrm{mcm}$ was observed on the interface copper-stainless steel generated by high-temperature rolling.

2) It was shown that changes of micro-hardness and nano-hardness relatively to the interface of two metals have common laws: less hard material-copper is hardening when approaching the interface of two metals and harder material-stainless steel is softening. This corresponds to the results of similar studies, performed on solid phase bonds $\mathrm{Cu}-\mathrm{Pb}$ and $\mathrm{Cu}-\mathrm{Nb}$, performed according to the same methods and discovered with deeper penetration of more fusible metal.

3) Structural studies witness the dislocation-stimulated diffusion of atoms of alloying elements from steel $08 \mathrm{X} 18 \mathrm{H} 10 \mathrm{~T}$ into copper during the high-temperature rolling in vacuum.

4) New mechanism of this process was discovered, and connected with collective transfer of atomic clusters consisting of add-mixtures and alloying elements.

\section{Conflicts of Interest}

The authors declare no conflicts of interest regarding the publication of this paper.

\section{References}

[1] Borts, B.V., Danilenko, N.I., Parkhomenko, A.A., Tkachenko, V.I., Korotkova, I.M., Vorobjev, I.A. and Lopata, A.A. (2014) Nano-Mechanisms of Deformation Close to the Interface of Dissimilar Materials Joined in Solid Phase by Means of High Temperature rolling. Open Journal of Metals, 4, 107-111.

[2] Borts, B.V., Parkhomenko, A.A., Vorobjev, V.A., Lopata, A.A., Aleksandrov, V.A. and Domnich, M.P. (2018) Changes in the Structure and Properties of the $\mathrm{Cu}-\mathrm{Pb}$ Composites Interface Jointed in the Solid Phase. Open Journal of Metals, 8, 55-65. https://doi.org/10.4236/ojmetal.2018.83004

[3] Borts, B.V. (2012) Formation of the Joint of Dissimilar Metals in the Solid Phase by the Method of Vacuum Hot Rolling. Materials Science, 47, 689-695.

https://doi.org/10.1007/s11003-012-9445-6

[4] Neklydov, I.M., Borts, B.V. and Tkachenko, V.I. (2011) Features of Formation of Dissimilar Metal Joints in Hot Roll Welding in Vacuum. Paton Welding Journal, 
No. 27, 27-32.

[5] Greenberg, B.A., Ivanov, M.A., Kuzmin, S.V. and Lysak, V.I. (2017) Explosive Welding: Processes and Structures. Innovation Enginnering, 235 p.

[6] Kuksenko, V., Pareige, C. and Pareige P. (2012) Intra Granular Precipitation and Grain Boundary Segregation under Neutron Irradiation in a Low Purity Fe-Cr Based Alloy. Journal of Nuclear Materials, 425, 125-129. https://doi.org/10.1016/j.jnucmat.2011.10.031

[7] Larikov L.N., Mazanko V.F. and Falchenko V.M. (1983) Mass-Transfer in Metals under Pulse Stressing. Physical and Mechanical Treatment of Materials, No. 6, 144-145.

[8] Petrov, Y.N., Falchenko, V.M., Mazanko, V.F., Yakubzev, I.A. and Vorona, S.P. (1988) Influence of Temperature on Nickel Atoms Mobility in the Austenitic Steel under High-Speed Deformation. Metal Physics, 10, 124-129.

[9] Zaharov, S.M., Larikov, L.N. and Mezhvinskii, R.L. (1995) Influence of Driving Force Created by External Pressure on Diffusion Mass-Transfer in the Solid Body. Physics of Metals and Advanced Technologies, No. 1, 30-35.

[10] Panin, V.E., Panin, A.V., Moiseenko, D.D., Shlyapin, A.D., Avraamov, U.S. and Koshkin, V.I. (2006) Physical Mesomechanics of a Deformed Solid as a Multilevel System. Physical Mesomechanics, 9, 5-13.

[11] Clayton, J.D., McDowell, D.L. and Bammann, D.J. (2006) Modeling Dislocations and Disclinations with Finite Micropolar Elastoplasticity. International Journal of Plasticity, 22, 210-256. https://doi.org/10.1016/j.ijplas.2004.12.001

[12] Borts, B.V., Danilenko, N.I., Necklyudov, I.M., Parkhomenko, A.A. and Firstov S.A. (2011) Nano-Structuring and Mass-Transfer Close to Materials Interface, Joint by Method of Hot Rolling in Vacuum. Metal Physics and Advanced Technologies, 33, 1035-1044.

[13] Konstantinova, T.E., Varukchin, V.N., Glasunova, V.A. and Burhovetskii, V.V. (2016) Defects in Nb-Ti Alloy, Deformed in Conditions of High Pressures. Polytechnic University, Saint Petersburg, 51-53. 